\title{
Cuckoo Search Algorithm Based On Local Optimization In The PID Parameter Optimization
}

\author{
Lei Luo ${ }^{1, a}$, Lixia Lv ${ }^{1, b}$ \\ ${ }^{1}$ School of Control and Computer Engineering, North China Electric Power \\ University,Baoding, 071000, China

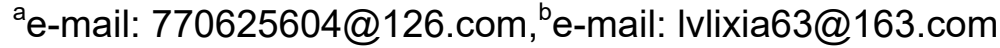

Keywords: cuckoo search algorithms; local optimization; PID parameter

\begin{abstract}
In order to solve the problem that the cuckoo search algorithm is easy to fall into local optimal value and cannot find global optimal value, propose improved cuckoo search algorithm based on local search optimization strategy. By extreme dynamic optimization algorithm local search operator is introduced into the latter part of the search cuckoo, make cuckoo search algorithm find that the global optimum value terms have better capabilities. Finally, the cuckoo algorithm applied to PID parameters optimization, and achieved satisfactory parameters.
\end{abstract}

\section{Introduction}

Cuckoo search algorithm is a new intelligent optimization algorithm proposed by yang et al., which has the advantage of fewer parameters, fast convergence speed and global search capability strong [1]. Some research results also prove that it has better performance than genetic algorithm, particle swarm algorithm and firefly algorithm [2]. This makes the cuckoo search algorithm has been widely applied to many fields, such as multi objective optimization [3], engineering design[4], neural network training [5], computer network[6].however, cuckoo search algorithm as a new intelligent optimization algorithm, the research is still in the initial stage, the global search ability and local optimization precision still need to improve. For example, the literature [8-10] by improving the levy flight search mechanism step, to improve the performance of search algorithm. In this paper, the performance of the algorithm is improved by hybrid of cuckoo search algorithm and PSO algorithm, and the simulation results show that the method is effective. The paper [10] the cuckoo search algorithm and the Powell search algorithm combination, put forward a Powell local search strategy based on global optimization cuckoo search algorithm. The paper [11] through the adaptive adjustment of step length factor to improve the cuckoo search algorithm of local and global search ability and convergence speed and experimental verification achieved better effect.

The improved algorithm improves the search performance of the algorithm to a certain extent, but it also increases the complexity of the algorithm in varying degrees. However, in addition, for some complex optimization problems and high dimensional search space, there are defects in the optimization ratio is not high.

Aiming at the defects of the cuckoo algorithm put forward a cuckoo algorithm based on local optimization operator. In the cuckoo search algorithm framework, the introduction of extremal dynamics search operator to improve the solution accuracy and convergence speed, and through a large number of numerical simulation experiments verify the validity. Compared the improved cuckoo search algorithm applied in PID parameters optimization, and simple method to obtain the optimal parameters. The experimental results show that, the improved cuckoo search algorithm to find the better, the PID controller to control object control effect best. And verify the effectiveness and applicability of the improved cuckoo search algorithm. 


\section{Cuckoo Search Algorithm And Its Improvement}

\section{The cuckoo algorithm}

Cuckoo search algorithm is optimization algorithm by cuckoo parasitism hatched education inspired by biological phenomenon of nestlings and design a mechanism based on levy flight search.

In nature, the cuckoo to find suitable for their spawning nest location is random or stochastic in a manner similar to, in order to simulate the cuckoo nest seeking; first of all, you need set the following three ideal states:

(1)The cuckoo produced only one egg at a time, and randomly selected nest to hatch it;

(2)In a group of randomly selected in the nest, the best nest will be reserved to the next generation;

(3)The number of nest available is fixed; the probability of nest owners can find an exotic bird egg. Based on these 3 ideal state on the path and position of the cuckoo WO update formula is as follows:

$$
\begin{aligned}
& X_{i}^{t+1}=X_{i}^{t}+\alpha \oplus \operatorname{Levy}(\lambda) \\
& \alpha=\alpha_{0}\left(X_{\mathrm{i}}^{\mathrm{t}}-X_{\text {best }}\right) \\
& \operatorname{Levy}(\lambda)=\frac{u}{|v|} \\
& \mathrm{u} \sim N\left(0, \delta_{U}^{2}\right) \\
& v \sim N(0,1) \\
& \delta_{\mathrm{u}}=\left\{\frac{\Gamma(\lambda) \sin (\pi(\lambda-1) / 2)}{\Gamma[(\lambda) / 2](\lambda-1) 2^{(\lambda-2) / 2}}\right\}^{1 /(\lambda-1)}
\end{aligned}
$$

In these formulas, $\mathrm{x}_{\mathrm{i}}=\left(\mathrm{x}_{\mathrm{i}}{ }^{1}, \mathrm{x}_{\mathrm{i}}{ }^{2}, \ldots \mathrm{X}_{\mathrm{i}}{ }^{\mathrm{n}}\right)(\mathrm{i}=1,2, \ldots$,$) , which mean the \mathrm{i}$-th nest position in the $\mathrm{t}$ generation, $\mathrm{n}$ mean the dimension optimization problem; $\mathrm{m}$ is the number of each generation of bird's nest; $\propto$ mean step size factor, to control the range of random search, a 0 as a constant, usually take 0.01 , Xbest represent the current optimal solution; $\square$ point to point multiplication; levy $(\lambda)$ is a random search vector which the parameter from 1 to 3 generated by the levy flight.

\section{Improved cuckoo algorithm}

The cuckoo algorithm based on levy flight search strategy in the search, has great randomness and to a certain extent, can prevent falling into a local optimum, but with the increase of the search area makes the algorithm accuracy decreased. In this paper, a local search algorithm is introduced into the cuckoo search algorithm, after a certain number of each iteration of a local search, to enhance the local optimization and improve the optimization performance. The search procedure of the local search algorithm is as follows:

(1)For the individual, set up the optimal solution to the xbest, whose objective function value is c (xbest).

(2)The individuals were the following: (a) fitness is calculated for each element of $x i j, j \in\{1,2, \ldots$ $\mathrm{N}$; ;(b) sort the fitness, find the smallest fitness groups, mean $\lambda \mathrm{i} \min \leqslant \lambda \mathrm{i} j, \mathrm{j}=1,2, \ldots \mathrm{n}, \mathrm{n}$ is the worst group; (c) in the neighborhood of the current individual to find a neighbor Xit, forced to make the worst set of elements to change;(d) unconditional acceptance Xit = Xit'; (e) if the current objective function value $\mathrm{c}(\mathrm{x})$ is less than the optimal objective function value $\mathrm{c}(\mathrm{xbest})$, $\mathrm{xbest}=\mathrm{Xit}$, $\mathrm{c}(\mathrm{xbest})=\mathrm{c}(\mathrm{Xit})$.

(3)Repeat steps (2) until the termination condition is met.

(4)Optimal solution xbest and objective function optimal value $\mathrm{c}$ (xbest) are obtained.

The search procedure of the improved cuckoo algorithm as follows: 
(1)Initialization algorithm data: set the number of bird grass $\mathrm{N}$, the maximum number of iterations maxcycle, the local search operator factors LN. Initialize the initial bird group $\mathrm{Xi0}, \mathrm{i}=(1,2, \ldots \mathrm{M})$,calculate the fitness of each bird swarm. If the local search operator factors equal zeros, and then execute local search procedure, after the completion of execution to a step (4); otherwise, continue to the next step.

(2)For each bird's nest according to levy flight to generate a new position, and calculate the fitness, if new explanation is better than the old solution, replaces old solution.

(3)The roulette method determines whether or not the host abandons the nest, and if it is abandoned, the other is to establish a new nest.

(4)Determine whether to meet the termination conditions, if not satisfied, then make $\mathrm{t}=\mathrm{t}+1, \mathrm{LN}=$ LN -1, turn step (2).

Standard test function

In order to verify the effectiveness of the CS-EO algorithm proposed in this paper, the numerical simulation experiments, we selected 6 test functions to illustrate the comparison, and compared with the cuckoo algorithm.

In the test, by ICSA and CSA algorithm respectively to 6 groups of test function of 30 tests, it were recorded in the running time of the algorithm and the computation of the optimal value of the mean value and variance; procedures were successful in finding the best success rate and the average number of iterations. In the table 1, NFES is the successful optimization of the average number of iterations, SR is the optimal success rate, NFES is the average number of iterations to find the success, SD is the 30 test of the variance, $t$ is the average running time, Mean is the optimal average value. Among them, computing the optimal value of the average value represents the program to find excellent performance is good or bad, variance and procedures were successful in finding the best success rate stability characterization algorithm; the running time of the algorithm and the average number of iterations characterization algorithm search optimization ability and to explore the optimal value of ability. The test results are as follows:

TABLE I TEST RESULTS

\begin{tabular}{|c|c|c|c|c|c|c|c|c|c|c|}
\hline \multirow{2}{*}{ Function name } & \multicolumn{5}{|c|}{ CS } & \multicolumn{5}{c|}{ CS-EO } \\
\cline { 2 - 12 } & NFES & SR & SD & T & Mean & NFES & SR & SD & T & Mean \\
\hline Michalewicz & 5577 & 0.9 & 0.0075 & 11.93 & -9.6586 & 3776 & 1 & $1.62 \mathrm{e}-5$ & 7.4165 & -9.66 \\
\hline Whitley & 32700 & 0.1 & 50.6973 & 74.508 & 57.3096 & 1139.3 & 1 & $1.49 \mathrm{e}-6$ & 20.22 & $8.64 \mathrm{e}-6$ \\
\hline Expansionf & 28713 & 0.37 & 0.9070 & 132.5 & 0.4379 & 17255 & 0.8 & 0.0047 & 114.9 & 0.0099 \\
\hline Rastrigin & 14383 & 0.43 & 1.361 & 36.57 & 1.1612 & 5034.2 & 1 & $7.99 \mathrm{e}-5$ & 8.38 & $9.14 \mathrm{e}-4$ \\
\hline Rosenbrock & 8759.6 & 0.93 & 0.9944 & 11.54 & 0.2659 & 8943.5 & 1 & $5.49 \mathrm{e}-6$ & 13.75 & $9.46 \mathrm{e}-5$ \\
\hline Schwefel & 14690 & 0.6 & 105.54 & 40.8 & -12491 & 13400 & 1 & 49.92 & 62.12 & -12542 \\
\hline
\end{tabular}

Through the test results in Table 1, ICSA in search depth, the optimization efficiency has been greatly improved, the optimization results, on average, ICSA algorithm has been significantly improved. The ICSA algorithm compared to CSA algorithm is improved, with better optimization ability and efficiency.

\section{Controlled Object And Control law}

\section{Controlled object}

In modern thermal power plant thermal control, boiler exit superheat steam temperature (main steam temperature) is one of the main parameters of boiler and also the whole steam trip refrigerants maximum temperature, to the power plant safe and economic operation has a significant impact. In general, temporary deviation of high-pressure boiler main steam temperature is not allowed to exceed 10 degrees, the long-term deviation does not exceed plus or minus 5 DEG c, this requirement is very high on the steam temperature control system. In this paper, a supercritical 
boiler 300mw thermal power unit main steam temperature control system as an example, and the results show that improved cuckoo search algorithm parameters optimization effect. The main steam temperature control system transfer function is ${ }^{[12]}$ :

Pre guiding area: $G(s)=\frac{0.93}{(73.3 s+1)^{2}}$

Inert zone area: $G(s)=\frac{1.94}{(88.5 s+1)^{6}}$

Objective evaluation function: $Q=\int\left[c_{1} t|e(t)|+c_{2}|u(t)|\right] d t$

Among them, $\mathrm{c} 1$ and $\mathrm{c} 2$ in order to adapt the evaluation coefficient function, related to the stable value and the output ratio of the proportion of vacant, can limit the output of the controller, so as to the maximum extent to simulate the control effect of the actual situation of the scene. And $\mathrm{c} 1=0.02, \mathrm{c} 2=0.98$ 。

\section{Controlled law}

At present, most of the industrial controllers are PID controllers. Because the control principle is simple, the parameters are few, and the control performance is stable. So the application scope is very wide. The PID parameters have experience tuning method, although more practical, but need several attempts, time-consuming and laborious, therefore how to use an intelligent algorithm to search automatically the parameters of the PID controller, become a trend of the future.

In this paper, the parameter optimization of cascade PID controller is carried out by ICSA algorithm. And in order to find the parameters into the cascade controller, the control effect of the cascade controller is observed, And with the basic particle swarm algorithm and basic cuckoo search algorithm for cascade PID parameter optimization method to optimize the control effect were compared, the control effect is as follows 。

The control effect is as follows:

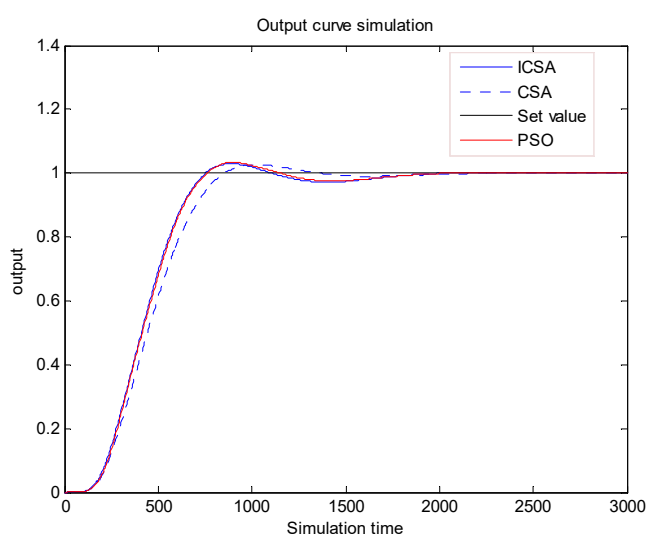

Fig 1 Output response

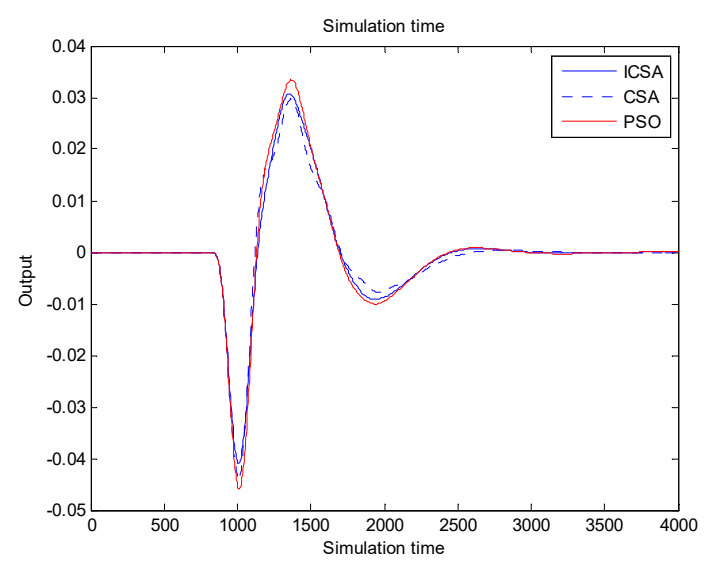

Fig 2 Interference response

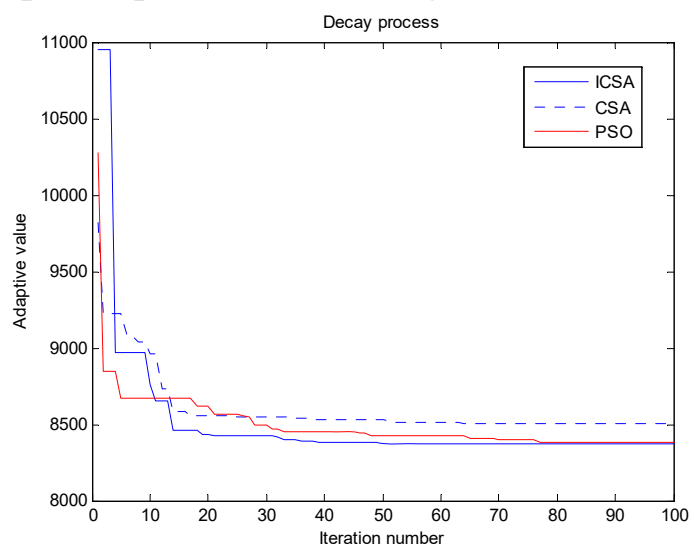

Fig 3 Attenuation curve 
As can be seen from the figure, in the step response, the cuckoo search algorithm to find the improvement PID parameters and particle swarm to find parameters for the controlled object order jump control than the cuckoo search algorithm to find the PID parameters control effect is better, compared the three output, PID controller by improved cuckoo algorithm response can quickly reach the set value. In the process disturbance, the performance, improved cuckoo strategy algorithm to find out the PID parameters of the anti-jamming effect are good. The output disturbance caused by the disturbance is smaller than that of the other two. In search efficiency, improved cuckoo search algorithm in finding the parameters in the process is very fast, and the PSO algorithm in the near optimal value when the searching efficiency decreased. While the cuckoo search algorithm to search the optimal value into a local optimum, and did not find the most suitable parameters of controller.

At run time, improved cuckoo search algorithm running 13.600522 seconds and cuckoo search algorithm running 33.955895 seconds. This shows improved cuckoo search algorithm effectively speed up the algorithm searching efficiency, find the optimum speed and computing time was shortened greatly, however, PSO algorithm is run 3.880548 seconds, illustrate the operation speed of particle swarm optimization algorithm the fastest. But due to the particle swarm optimization algorithm with high efficiency without cuckoo search algorithm, it also needs to be improved.

\section{Concluding Remarks}

The cuckoo search algorithm in the presence of accuracy for low, it is easy to fall into local optimum points, proposed a new local search of the cuckoo search algorithm based on. The algorithm makes full use of the local search algorithm to improve the local searching capability of the cuckoo algorithm. Finally will three algorithms are used on the cascade PID controller parameters optimization. The simulation results show that improved cuckoo search algorithm in parameter global searching ability, convergence speed and solution precision were is superior to that of the former two methods. Therefore, improved cuckoo search algorithm is proposed in this paper can become an effective parameter optimization strategy.

\section{Acknowledgment}

This project was supported by State Key Laboratory of Alternate Electrical Power System with Renewable Energy Sources. Thanks a lot.

\section{References}

[1] YANG X S, DEB S. Cuckoo search via Lévy Flights[C]//nabic 2009: Proceedings of World Congress on Nature\&Biologically Inspired Computing. Coimbatore, India, IEEE Publications, 2009: 210-214.

[2] Liu Changping, ye Chun Ming. The cuckoo algorithm for permutation flow shop scheduling problem[J].Journal of University of Shanghai for Science and Technology, 2013, 35 (1): 17-20.

[3] YANG X S, DEB S. Multi-Objective Cuckoo Search for Design Optimization[J].Computers \& Operations Research, 2013,40(6):1616-1624.

[4] Chen yue. Engineering structural optimization problem solving modified cuckoo search algorithm [J]. Computer application research, 2014,31 (3): 679-683.

[5] Valian E. Improved cuckoo search algorithm for feedforwar neural network training[J]. International Journal of Artificial I\&A, 2011,2(3):36-43.

[6] LAI Jinhui. Application of GCS-SVM model in network traffic prediction[J]. Computer and Application, 2013, 49(21):75-78. 
[7] Lan Shaofeng, Liu Sheng. Review of cuckoo search algorithm[J]. Computer engineering and design, 2015,36 (4): 1603-1607.

[8] TUBA M, SUBOTIC M, STANAREVIC N. Modified Cuckoo Search Algorithm for Unconstrained Optimization Problems[C]//Proceedings of the 5th European Conference on Computing. Athens, Greece, 2011: 263-268..

[9] GHODRATI A, Lotfi S. A Hybrid CS/PSO Algorithm for Global Optimization[C]//Proceedings of the 4th Asian Conference on Intelligence information and Database Systems. Kaohsiung, China, 2012: 89-98.

[10] Ma Wei, Sun Zhengxing, Li Jun. Building Powell local search strategy for global optimization algorithm based on the cuckoo[J] Computer application research, 2015,32 (6): 1667 -1675.

[11] Qian Weiyi, Hou Huichao, Jiang Shouyong. A new cuckoo bird adaptive search algorithm[J]. Computer science, 2014,41 (7): 279 -282.

[12] Han PU. Intelligent control theory and its application [M]. China Electric Power Press.2012, 12. 